\title{
Estrategias de aprendizaje de universitarios en español como lengua extranjera para el sector profesional: estudio de caso en Alemania
}

\author{
María del Carmen Mahúgo Cárdenes \\ Universidad de Las Palmas de Gran Canaria \\ Ansbach University of Applied Sciences \\ mmahugo@yahoo.es
}

\section{Resumen}

En el contexto europeo de enseñanza superior, el aprendizaje de una lengua extranjera (LE) es fundamental para el desarrollo de los futuros profesionales. Este estudio de caso analiza las estrategias de aprendizaje de lengua (EAL) que utilizan estudiantes de español como lengua extranjera (ELE) en Alemania. Para este estudio se ha utilizado como herramienta fundamental el cuestionario de estrategias SILLStrategy Inventory for Language Learning (OXFORD, 1990) en diferentes universidades del estado de Baviera. Los resultados indicaron que: las estrategias social y compensatoria son las más utilizadas; las mujeres utilizan en su mayoría las categorías mnemotécnicas y compensatorias mientras que los hombres emplean las metacognitivas y cognitivas; y la variable de LE influye en la frecuencia de uso de la categoría compensatoria. Estos resultados esperan ser una pequeña contribución al conocimiento de EAL de los aprendientes de ELE europeos.

Palabras clave: contexto europeo; enseñanza superior; EAL; ELE

\begin{abstract}
In the European context of higher education, learning a foreign language (FL) is essential for the development of future professionals. This study of case analyzes the language learning strategies (LLS) used students of Spanish as a foreign language (SFL) in Germany. For this study, the strategy questionnaire SILL-Strategy Inventory for Language Learning (OXFORD, 1990) has been used as a fundamental tool in different universities of the state of Bavaria. The results indicated that: social and compensatory strategies are the most used; women use memory and compensatory strategies mostly, while men use metacognitive and cognitive strategies; and the FL variable influences the frequency of use of the compensatory category. These results hope to be a small contribution to the LLS knowledge of European SFL learners.
\end{abstract}

Keywords: European context; Higher Education; LLS; SFL 
Estrategias de aprendizaje de universitarios en ELE para el sector profesional...

\section{Introducción}

Aprender un idioma extranjero forma parte de la formación transversal de cualquier profesional en el espacio europeo. De hecho, según la libre circulación de trabajadores es un principio fundamental establecido en el artículo 45 del Tratado de Funcionamiento de la Unión Europea. Cada vez más, en los centros universitarios europeos se impone el aprendizaje de otras lenguas como requisito indispensable para completar una amplísima gama de estudios, ya no sólo de carácter internacional. Por lo tanto, la evolución en el aprendizaje de lenguas nos hace recapitular e insistir en crear nuevos métodos y técnicas de aprendizaje, y mejorar los existentes.

Este hecho hace de vital importancia el análisis constante de las necesidades de los universitarios y por consiguiente el análisis en sí del propio estudiante. Conocer el perfil de nuestros estudiantes, cómo piensan, cómo actúan con todo el ambiente tecnológico que los rodea es imprescindible para avanzar en nuestra meta como profesores de una segunda lengua o lengua extranjera (L2 o LE) ${ }^{1}$.

Según Cáceres Lorenzo (2014, p. 229):

La transcendencia que tiene para el universitario el progreso de una conciencia metalingüistica (language awareness) se debe entender como el desarrollo del conocimiento explícito de la lengua y del saber aprender (autonomía del alumno) de la competencia discursiva.

Aprender una LE depende también de las necesidades e intereses particulares de cada estudiante y este proceso de aprendizaje de la lengua meta conlleva una responsabilidad que el aprendiz de idiomas debe asumir desde un primer momento. Debemos así considerar que el estudiante podrá adquirir la LE a través de un aprendizaje autónomo que creemos debe ser objetivo primordial en la enseñanza/aprendizaje de la LE. Feld-Knapp (2010, p. 21) lo denomina así:

${ }^{1}$ Segunda lengua/lengua extranjera: Se diferencia entre estos dos términos para resaltar que, en el primer caso, se trata de una lengua hablada en la comunidad en que se vive, aunque no sea la lengua materna del aprendiz, mientras que, en el segundo caso, la lengua no tiene presencia en la comunidad en la que vive el aprendiz. (MUÑOZ, 2002, p. 112-113) 
Con el concepto de "aprendizaje autónomo" se denomina una cultura de aprendizaje que se centra en la conexión con experiencias personales y motivos de aprendizaje, así como en el desarrollo de habilidades para el aprendizaje independiente. Para un aprendizaje independiente que no cuente con el apoyo del profesor, se necesitan estrategias y planes de acción mental que se usen conscientemente $y$ de manera específica durante el aprendizaje. $^{2}$

Sin embargo, Fernández-Castillo (2015) advierte que son muchos los interrogantes que giran todavía en torno a este tema, y los procesos de aprendizaje que diferencian a unos estudiantes de otros se deben tanto a los estilos de aprendizaje como a los éxitos y los fracasos personales en el aprendizaje de cada estudiante. Asimismo, en el proceso de aprendizaje incurren factores personales que, a priori, no se pueden obviar, como la edad, el género, el nivel cultural, la procedencia, la motivación, incluso la personalidad, como menciona García Herrero (2013), además, existe un amplio consenso entre los expertos que coinciden en la repercusión de dichos factores.

De este modo, nuestro trabajo se ha interesado por el problema de investigación dentro del ámbito de la Linguística Aplicada que se centra en el proceso de enseñanza/aprendizaje de una lengua extranjera en el contexto europeo, en el que se integra, en este caso, el sistema universitario alemán. Este sistema está involucrado en un cambio de paradigma, en el cual el enfoque deja de centrarse en el docente para hacerlo en los estudiantes, así como en las decisiones autónomas que ellos toman en el momento de asumir una determinada tarea y utilizar una estrategia de aprendizaje, como Bimmel apunta (2012, p. 4) "una estrategia de aprendizaje es un plan mental para alcanzar una meta de aprendizaje". 3

\footnotetext{
${ }^{2}$ Mit dem Begriff des a.L. wird eine Lernkultur bezeichnet, in der das Anknüpfen an die persönlichen Erfahrungen und Lernmotive sowie die Entwicklung der Fähigkeiten für ein eigenverantwortliches Lernen im Zentrum stehen. Für das selbstständige, von der Lehrkraft unabhängige Lernen sind Strategien, mentale Handlungspläne notwendig, die beim Lernen bewusst und zielgerichtet eingesetzt werden. (FELD-KNAPP, 2010, p. 21)

${ }^{3}$ Eine Lernstrategie wäre dann ein Handlungsplan, um dieses Lernziel zu erreichen (BIMMEL, 2012, p. 4)
} 
Partiendo de este contexto europeo se ha indagado sobre las variables que presenta el alumnado universitario y la influencia de dichas variables en la adopción de un tipo de estrategia de aprendizaje, ya que como hemos explicado, esto puede afectar de manera significativa a la adquisición de la lengua meta. Creemos que las competencias lingüísticas en universitarios que aprenden español como lengua extranjera (ELE) se desarrollan si convertimos a los alumnos en aprendientes autónomos, por consiguiente, es necesario no sólo el análisis de este proceso de enseñanza/aprendizaje sino también averiguar qué factores personales tienen impacto en el proceso de adquisición de otra lengua.

En consecuencia y partiendo de la necesidad existente de enseñar el español como LE dentro del contexto europeo, el objetivo de este estudio es analizar y definir las estrategias de aprendizaje de ELE y los factores personales que influyen en la elección de las mismas. Al mismo tiempo, nos basamos en el hecho de que para poder obtener un conocimiento implícito y explícito de la lengua es imprescindible llevar a cabo el uso de estrategias de aprendizaje.

Con los resultados de esta investigación nos proponemos aportar una contribución al conocimiento de Estrategias de aprendizaje de lengua (EAL) de los aprendientes de LE europeos y ayudar a mejorar las habilidades lingüísticas de los alumnos en el aprendizaje de la lengua meta y el necesario desarrollo de su independencia. En este sentido, Fernández-Castillo (2015) apunta que "el entrenamiento específico de estrategias se asocia, a que los estudiantes las incorporen de forma efectiva y eficiente a su sistema de trabajo a lo largo de la adquisición de la L2, a un mayor uso social, e incluso en contextos naturales".

En esta investigación hemos realizado un análisis de la frecuencia de uso de las estrategias de aprendizaje y la posible relación entre las variables personales y dicha frecuencia. A través de los datos que arrojan los resultados estadísticos realizados, queremos dar a conocer la información obtenida, la cual, nos permitirá responder a las siguientes preguntas de investigación que hemos planteado para este estudio de caso:

(1) ¿Cómo se definen las estrategias de aprendizaje de los estudiantes universitarios de ELE en Alemania? Desde el punto de vista del contexto europeo tratamos de definir el tipo de estrategia o estrategias que representa el paradigma de estudio de la lengua española como lengua extranjera en un país europeo.

(2) ¿Cuál o cuáles son las estrategias de aprendizaje predominantes? 
¿Existen una o varias estrategias predominantes en cada una de las universidades en las que se ha llevado a cabo este estudio? Analizamos desde el punto de vista de la lingüística aplicada el reconocimiento de las distintas estrategias dentro de los grupos de estudiantes seleccionados y las contrastamos.

(3) ¿Existe alguna variable personal que influya en el uso de las estrategias?

En cada uno de los análisis hemos recopilado y contrastado los datos referentes a las variables de edad, sexo, L1 y L2 con las diferentes estrategias de aprendizaje.

\section{Marco teórico}

\subsection{Descripción de estrategias de aprendizaje}

Desde los comienzos de la enseñanza de lenguas extranjeras se han realizado múltiples investigaciones para estudiar el tipo de estrategias que utilizan los aprendientes durante el proceso de aprendizaje de una LE. En este proceso, el aprendiz pone en marcha una serie de mecanismos o estrategias de aprendizaje que están formadas por secuencias de distintas actividades que cada aprendiz planifica por iniciativa propia, y de manera deliberada y autónoma. Estas estrategias de aprendizaje son de vital importancia para la adquisición de la lengua meta ya que a través de ellas se desarrolla y completa el proceso de aprendizaje.

Para O'Malley y Chamot (1990) las estrategias de aprendizaje son pensamientos o comportamientos que los individuos usan para ayudarse a comprender, aprender o retener nueva información. Cohen (1990) afirma que sólo forman parte de los procesos cognitivos del aprendizaje y según Griffiths (2008) son actividades seleccionadas conscientemente por los aprendientes para regular su propio aprendizaje de idiomas. Oxford (1990), por su parte, las define como comportamientos o acciones usadas por los estudiantes para hacer el aprendizaje de idiomas más exitoso, autodirigido y agradable. Todos estos autores coinciden en que las estrategias de aprendizaje forman parte de un proceso individual.

El Instituto Cervantes expone que: 
Estrategias de aprendizaje de universitarios en ELE para el sector profesional...

No existe unanimidad de criterios sobre el concepto y su definición, puesto que las estrategias de aprendizaje constituyen un vasto conglomerado de técnicas, habilidades, procedimientos $y$ destrezas. Las hay conscientes e inconscientes, innatas o adquiridas, observables y no observables, etc.

Tras estudiar las investigaciones y clasificaciones acerca de las estrategias de aprendizaje de una LE de autores de prestigio como los mencionados anteriormente, hemos decidido seguir el concepto $y$ clasificación de Oxford (1990). Así mismo, se ha utilizado el inventario SILL-Strategy Inventory for Language Learning (OXFORD, 1990) como instrumento para medir las estrategias, por lo tanto, seguiremos la clasificación desarrollada por dicha autora. Según las investigaciones de Oxford (1990) sobre las estrategias de aprendizaje, estas pueden ser clasificadas en dos categorías diferentes: directas e indirectas.

Las estrategias directas tienen que ver con el procesamiento mental del aprendiente y se subdividen a su vez en tres categorías: cognitivas, mnemotécnicas y de compensación. Las estrategias cognitivas son aquellas con las que el aprendiz puede manipular las estructuras de la propia lengua mediante el razonamiento y el análisis del aprendizaje de la LE. Las mnemotécnicas ayudan al estudiante a relacionar términos con la LE sin que tengan que ser necesariamente comprendidos y esto significa no solo memorizar vocabulario, sino también expresiones lingüísticas y estructuras gramaticales. Las de compensación estimulan las habilidades comunicativas ya que el hablante debe utilizar sinónimos, muletillas, interjecciones o incluso preguntar para poder continuar con la comunicación.

Las estrategias indirectas son también necesarias ya que complementan el aprendizaje de la LE y se clasifican en metacognitivas, afectivas y sociales. Las metacognitivas permiten reflexionar sobre el propio proceso de aprendizaje, lo cual es muy positivo para dicho aprendizaje, aunque para ello sería de gran ayuda recibir una educación en la organización y planificación del estudio de una LE. Las afectivas sirven para controlar y modificar la actitud frente al aprendizaje, por ejemplo, situaciones de estrés y frustración pueden ser muy negativas para el proceso de aprendizaje e identificarlas y controlarlas es importante para el aprendiz. Finalmente, las estrategias sociales potencian las interacciones entre los aprendientes, por ejemplo, al estudiar conjuntamente e intercambiar dudas o practicar lo aprendido. 


\subsection{El aprendizaje de lenguas en Alemania}

En este estudio nos hemos centrado en el contexto del EEES (Espacio Europeo de Educación Superior) para observar los cambios que se están produciendo en la actualidad en la enseñanza/aprendizaje de lenguas extranjeras en el ámbito de la enseñanza superior, la cual se ha ido desarrollando con el fin de homogeneizar las políticas de regulación de la misma que exige el Plan de Bolonia en el Espacio Europeo (FRAILE ARANDA, 2006).

En el caso del trabajo que nos ocupa, hemos realizado un estudio de las estrategias de aprendizaje de estudiantes de español como lengua extranjera (ELE) en Alemania. En lo que respecta a Alemania, debemos mencionar que al contrario que otros países, siempre ha tenido un sistema educativo igualitario y con un alto nivel académico en general ${ }^{4}$. A partir de los diez años, se separa a los niños en tres tipos de colegio distintos (Gymnasium, Realschule y Hauptschule) según el nivel que hayan obtenido en sus primeros años educativos. Para acceder a la universidad, los estudiantes de secundaria tienen que hacer un examen de selectividad (Abitur) aunque la mayoría de los niños opta por unas prácticas (Lehre), que es un método existente sólo en Alemania para combinar práctica con formación académica adicional en una escuela de formación profesional (Berufsschule) o universidad. ${ }^{5}$

En España, el sistema educativo es diferente, pero los métodos de aprendizaje utilizados por los alumnos españoles de una LE guardan similitudes con los métodos del sistema educativo alemán, ya que al igual que en Alemania, estas estrategias comenzaron a desarrollarse con la posibilidad de aprendizaje de otras lenguas como el inglés o el francés en colegios y universidades. Ahora bien, en el sistema educativo español el desarrollo de las estrategias de aprendizaje de una LE ha sido más pausado, ya que, hasta hace relativamente poco tiempo no se empleaba un método comunicativo en las aulas. Esto, sin embargo, está cambiando notablemente en la actualidad, gracias al ingreso de España en la Unión Europea y a la globalización, como afirma Muñoz Zayas (2013).

\footnotetext{
${ }^{4}$ Informe del programa internacional para la Evaluación de Estudiantes o Informe PISA (Programme for International Student Assessment).

${ }^{5}$ Das Deutsche Institut für Internationale Pädagogische Forschung (DIPF).
} 
Según un estudio de Ignacio Olmos Serrano (2006), director del Instituto Cervantes de Berlín, las principales causas de este constante crecimiento del español en Alemania son la importante presencia internacional del español y el uso no solo turístico, sino también profesional de esta lengua. Por este motivo, muchas carreras de las universidades alemanas, no solo en el ámbito de la formación del profesorado de español, sino también de Economía y Empresariales, incluyen el español en el plan curricular de sus estudios.

Por otro lado, a pesar de la numerosa población de origen turco que posee Alemania, son muy pocos los estudiantes universitarios de este origen, siendo la mayoría de origen alemán y el resto procedentes de países del Este como Rumanía, Rusia y Polonia. A menudo nos encontramos con el caso de alumnos que han nacido en Alemania y sus padres son inmigrantes, a veces incluso de países diferentes, por lo que esos alumnos pueden poseer una L3 y hasta una L4, además de las lenguas extranjeras que más tarde aprenden en el colegio. Por ejemplo, un alumno nacido en Alemania de madre rusa y padre serbio puede comunicarse como hablante nativo en estos idiomas, además de hablar alemán por haber crecido y estudiado en el país y aprender en el colegio e instituto inglés, francés y/o español. El hecho de que los habitantes procedentes de otros países creen pequeñas colonias dentro de Alemania, algo que es visible en los barrios de muchas ciudades de Alemania, hace que esos niños sigan en contacto con la lengua materna de sus padres.

La importancia que se le da al conocimiento de una LE en España es menor que en muchos otros países de Europa tal y como apunta Lasagabaster (2012). Según el mismo autor, la mayoría de las universidades españolas aún carecen de una política que establezca los objetivos lingüísticos requeridos para que el "multilingüismo" sea una realidad en España, concretamente entre los estudiantes universitarios. Sin embargo, en Alemania, país en el que el interés por las lenguas extranjeras era bastante notable ya desde el siglo XIX, la tendencia a aprender otros idiomas es cada vez mayor, siendo el inglés la LE más importante en su sistema educativo.

De acuerdo con Alcalde Mato (2010, p. 8-29):

Alemania siempre se ha destacado no sólo por su carácter innovador, al introducir nuevos planteamientos metodológicos, ni por su significativa preocupación crítica para la mejora de los mismos, sino también por el interés creciente en este país por 
aprender idiomas extranjeros, independientemente de las escuelas y universidades, así como por ser especialmente sobresaliente en la publicación de materiales docentes, razones todas ellas más que suficientes para reconocerle una singular importancia en el campo de la enseñanza y aprendizaje de lenguas extranjeras.

Alcalde Mato (2011) explica que después de la Segunda Guerra Mundial, se utilizaba en Alemania el método de aprendizaje deductivo basado en la gramática y la traducción y que ya en la década de los 90 se empieza a notar la presencia de métodos muy variados y que se interesaban más en la comunicación. Esta fundamentación histórica sobre los métodos de aprendizaje utilizados en Alemania muestra que dichos métodos son una de las principales influencias para las estrategias de aprendizaje de los alumnos.

Grünewald (2009) realizó un estudio muy interesante sobre la motivación de los alumnos en la clase de lengua extranjera utilizando ordenadores como método de aprendizaje y así poder analizar su impacto. Este estudio mostró que los sujetos no sólo disponían de "un elevado potencial de reflexión acerca de su propio proceso de aprendizaje" (GRÜNEWALD, 2009, p. 85), sino que también les permitió "realizar afirmaciones válidas sobre sus propias estrategias de aprendizaje y sobre la organización de su proceso de aprendizaje de una lengua extranjera" (GRÜNEWALD, 2009, p. 86). Además de esto afirma que el uso de las tecnologías en la clase de LE "posibilita una organización del proceso de aprendizaje parcialmente autónomo, además de un trabajo independiente del profesor" y constata también que "la autogestión del alumno también es sinónimo de individualización del aprendizaje" (GRÜNEWALD, 2009, p. 86).

\subsection{Las variables}

En cuanto a las variables, hemos observado dos grandes factores externos como la edad y el sexo. La edad es un factor importante a la hora de adquirir una LE, siendo los adolescentes y los adultos jóvenes los más rápidos en el aprendizaje lingüístico ya que disponen de mayores capacidades cognitivas para aprender sobre la lengua y menos restricciones biológicas como el envejecimiento neuronal (MUÑOZ, 2002). En cuanto al sexo, algunos estudios afirman que las mujeres usan una mayor cantidad de 
estrategias que los hombres (OXFORD, 1990; EHRMAN y OXFORD, 1990; GREEN y OXFORD, 1995; GARCÍA HERRERO, 2012).

Por otro lado, y de acuerdo con Galindo Merino (2012), los procesos de aprendizaje de una LE son similares a los procesos de aprendizaje de una lengua materna (L1) y parte del éxito que pueda tener el estudiante a la hora de aprender la LE se basa en un buen dominio y conocimiento de la L1. Además de esto, se dan diferentes circunstancias dentro de este tema, por ejemplo, los estudiantes que desconocen los términos que definen los diferentes componentes y estructuras de una lengua pueden presentar una mayor dificultad al adquirir la LE, por otro lado, también la influencia de la L1 puede afectar al desarrollo del aprendizaje de la LE en el plano fonológico por ejemplo y otra circunstancia podría ser:

El impacto de la distancia interlingüística entre L1 y L2 en los cambios de código que se producen en el aula. Por ejemplo, para un hispanohablante no es lo mismo aprender chino que portugués, y esta distancia entre los idiomas tiene un claro efecto en la alternancia lingüística, como se adivina en los estudios sobre inglés como lengua extranjera en el contexto asiático. (GALINDO MERINO, 2012, p. 171)

Para Fernández-Castillo (2015, p. 402):

Aunque los individuos parecen adquirir la lengua materna incorporando elementos gramaticales sin necesidad de una programación estructurada e instruccional, sin una aparente planificación prediseñada y a lo largo de un proceso interactivo eminentemente natural de asimilación semántico-funcional, no parece ocurrir lo mismo cuando se enfrentan a procesos de adquisición de una segunda lengua.

Sánchez Castro (2008, p. 9), en su estudio sobre las dificultades de los hablantes nativos de alemán en el aprendizaje de los tiempos de pasado, sugiere que la influencia de la L1 es la principal causa de fracaso en el aprendizaje del español como lengua extranjera y concluye haciéndonos esta pregunta "¿por qué no servirnos de los conocimientos y estrategias que el estudiante ya posee en el aprendizaje de lenguas extranjeras para acelerar su aprendizaje del español?’. 
También existen otras variables que pueden influenciar el aprendizaje de la LE: los rasgos de la personalidad, la importancia de factores ambientales de carácter lingüístico como haber estudiado otras lenguas, factores sociolingüísticos como la importancia en la sociedad de la lengua meta, factores familiares, personales, pedagógicos e incluso los rasgos de la personalidad (RONCEL, 2005).

García Cobo (2013, p. 37) afirma en su estudio que "existe una relación directa entre el interés hacia los idiomas y la actitud positiva hacia ellos. Lo cual, sin duda, propicia la aparición de una motivación en el alumno que le impulsa a aprenderlo".

\section{Metodología}

Los estudiantes universitarios que han participado en este análisis forman un total de 251 alumnos de edades comprendidas entre los 21 y los 23 años y en su mayoría de nacionalidad alemana. Es importante aquí mencionar que, dentro del número de sujetos estudiados, el número de mujeres (161) supera al de hombres (90). Esto puede deberse al azar o a un interés mayor por parte de las mujeres en el aprendizaje de lenguas, sin embargo, no hemos investigado este dato para este artículo.

Los estudios que cursan los sujetos en las respectivas universidades son de una amplia variedad: Periodismo, Derecho, carreras técnicas de diferentes áreas de Ingeniería y Tecnología, estudios relacionados con las empresas, Trabajo Social, Diseño, Educación y Ciencias Naturales. Los sujetos estudiados cursan la asignatura de español de manera optativa en la mayoría de los casos y poseen, según el Marco común europeo de referencia (MCER), distintos niveles de competencia lingüística (A1, A2, B1, B2 y C1).

Como ya hemos mencionado con anterioridad, en los últimos 10 años se ha empezado a insertar el español como LE en los institutos del estado de Baviera y en Alemania en general, por lo que, en la actualidad se está incrementando el número de alumnos que escogen el español en el instituto. Como resultado de ello, cada vez hay una mayor cantidad de alumnos procedentes de institutos que llegan a la universidad con un nivel B1 de español. Por este motivo, aquellos que poseen un nivel superior de español asisten a cursos de A2 y B1 con el fin de repasar, de no olvidar el idioma y de obtener créditos extra para sus estudios. 
De los estudiantes seleccionados, 64 proceden de la Universidad de Ciencias Aplicadas de Ansbach (HS Ansbach), 70 de la Universidad de Ciencias Aplicadas de Núremberg Georg Simon Ohm (THN), 36 de la Universidad de Bayreuth y 81 de la Universidad Friedrich-Alexander de Erlangen-Núremberg (FAU), todas situadas en el estado de Baviera.

Como instrumento para recabar la información que precisamos para nuestro estudio hemos utilizado un cuestionario individual y escrito. Se trata de un cuestionario ya existente, acreditado para evaluar estrategias de aprendizaje, llamado SILL, del inglés Strategy Inventory for Language Learning (OXFORD, 1990). Este cuestionario es una herramienta que está creada y diseñada para analizar cómo se adquiere una LE a través de un inventario de estrategias.

El cuestionario consta de dos apartados: un primer apartado elaborado por nosotros y que contiene preguntas personales sobre la edad, el sexo y nivel de competencia lingüística de español y otros idiomas; y un segundo apartado que reúne las seis categorías de estrategias de aprendizaje según el SILL. En la adaptación que hemos realizado de la versión original, se ha mantenido el contenido de los 50 ítems del SILL que hacen referencia a las seis dimensiones (mnemotécnica, cognitiva, compensatoria, metacognitiva, afectiva y social) que engloban dichas estrategias de aprendizaje.

Para llevar a cabo el procedimiento hemos realizado en primer lugar una traducción y adaptación al alemán del cuestionario (Apéndice 1) cuya versión original es en inglés. Hemos intentado que esté expresado de forma clara y se refiera al tipo de tarea al que se hace referencia. Aunque las universidades tienen un gran número de estudiantes extranjeros, es necesario poseer un nivel $\mathrm{C} 1$ de alemán para estudiar en una universidad alemana por lo que se parte de que es esta la principal vía de comunicación de estos estudiantes. Por ello, se consideró oportuno realizar la traducción del cuestionario utilizado, ya que, de esta manera los cuestionarios aportan una mayor fidelidad. En segundo lugar y una vez terminada la elaboración del cuestionario, se procedió a su utilización con los sujetos de las cuatro universidades nombradas. El cuestionario se repartía y se completaba durante las clases de español. Esta tarea se llevó a cabo a lo largo de un semestre.

En cuanto al análisis estadístico de los datos obtenidos, se ha utilizado en primer lugar el mismo procedimiento de SILL, es decir, un análisis factorial. Aplicando este tipo de análisis calculamos las sumas de 
las diferentes partes de SILL y las hemos dividido entre el total de cada parte para obtener así un promedio general, que debe estar dentro del rango de 1 a 5. Las diferentes estrategias han sido puntuadas en relación a la frecuencia de uso de las mismas, por lo que les hemos asignado valores entre 1 (nunca); 2 (poco frecuente); 3 (frecuente); 4 (muy frecuente); y 5 (siempre). Para llevar a cabo su análisis en el programa SPSS se han asignado consonantes para los nombres de cada estrategia: A: Mnemotécnica; B: Cognitiva; C: Compensatoria; D: Metacognitiva; E: Afectiva; y F: Social.

Tras introducir estos cálculos y el resto de datos recopilados de las variables que sometimos a dicho procedimiento, hemos procedido al análisis estadístico desde un enfoque metodológico cuantitativo utilizando el programa estadístico SPSS 14.0 para Windows. Con este programa se realizaron análisis de la frecuencia de uso de las estrategias de aprendizajes, un T-Test a partir de una variable independiente para averiguar la diferencia de uso de estrategias con relación a la variable sexo y un test de correlaciones según Pearson para buscar las posibles influencias de las variables Edad, L1 y L2 sobre la frecuencia de uso de las estrategias de aprendizaje. Además, hemos realizado una comparación de medias y para ello hemos empleado ANOVA de un factor, ya que queremos saber si las medias de una variable son diferentes entre los niveles o grupos de otra variable. Con este tipo de análisis estadístico, ANOVA de un factor, hemos determinado la relación entre dos variables examinando si las medias de la variable dependiente son diferentes en las categorías o grupos de la variable independiente.

\section{Análisis y discusión de los resultados}

A continuación, presentamos las figuras y tablas informativas resultantes del proceso de análisis estadístico.

En las siguientes figuras mostramos una serie de gráficos de columnas que representan la frecuencia de uso de estrategias en cada una de las cuatro universidades (figuras 1-4) y la diferencia entre hombres y mujeres (figuras 5-8). De esta forma, podemos además comparar los valores resultantes entre las distintas universidades.

Las siguientes figuras muestran la puntuación obtenida en cada una de las dimensiones de las estrategias de aprendizaje: 
Estrategias de aprendizaje de universitarios en ELE para el sector profesional...

Figura 1. Estadístico descriptivo de frecuencia de uso de las categorías (HS Ansbach).

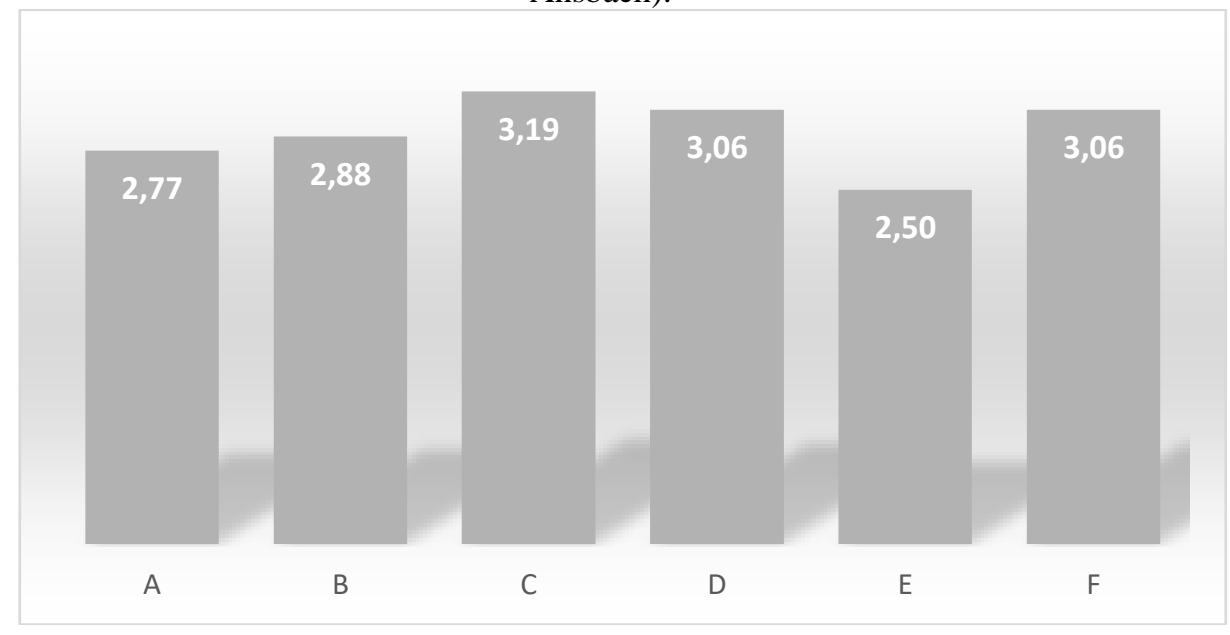

Figura 2. Estadístico descriptivo de frecuencia de uso de las categorías (THN).

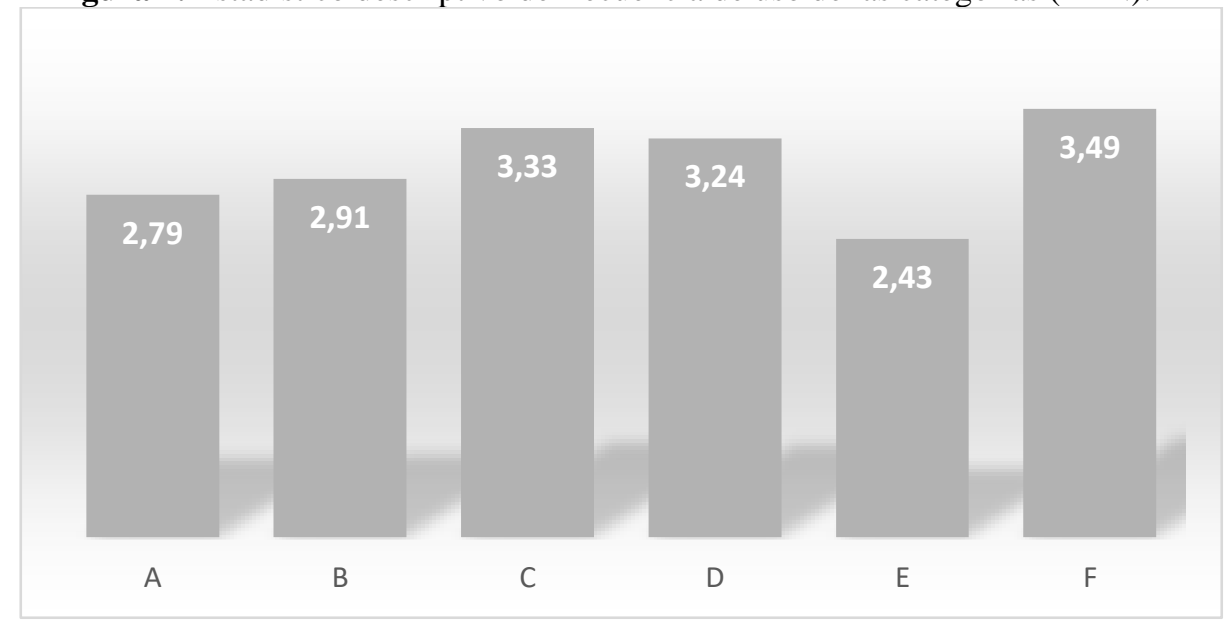


María del Carmen Mahúgo Cárdenes

Figura 3. Estadístico descriptivo de frecuencia de uso de las categorías (Universidad de Bayreuth).

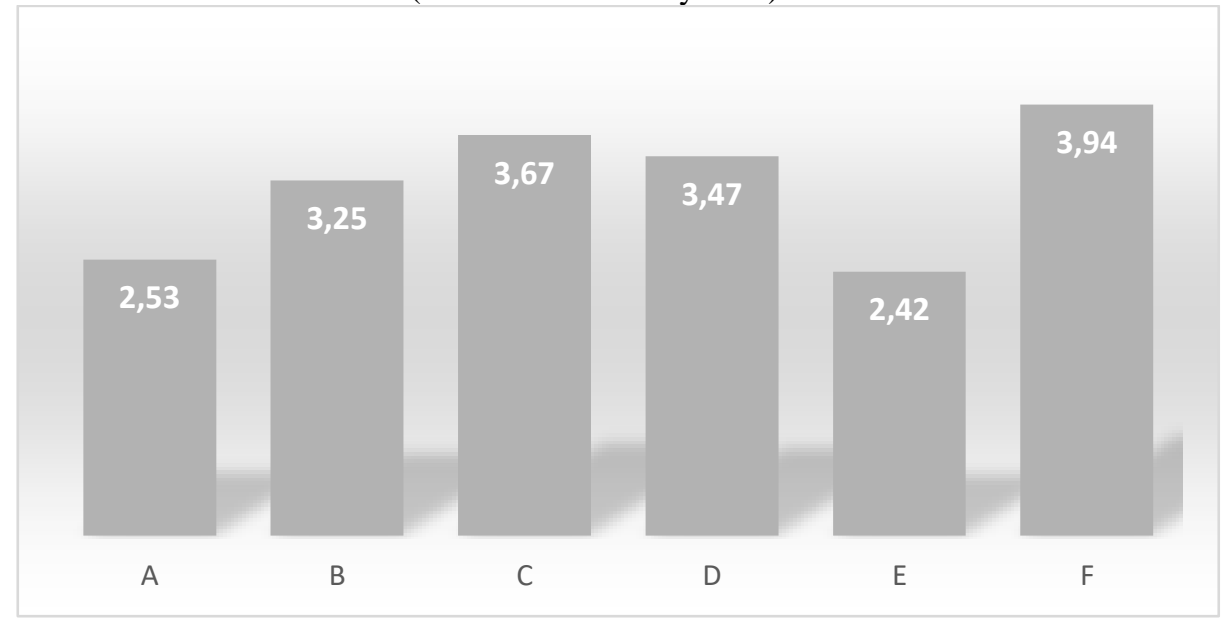

Figura 4. Estadístico descriptivo de frecuencia de uso de las categorías (FAU).

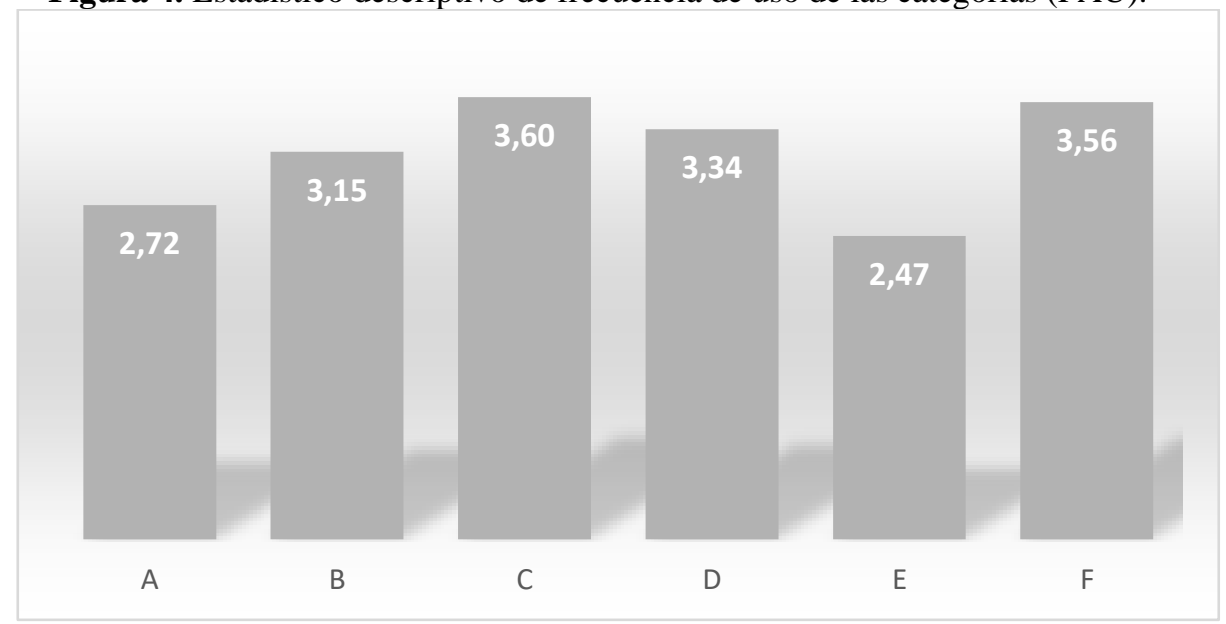

En la figura 1, el gráfico de barras revela que la estrategia compensatoria alcanza el valor medio más alto y la estrategia afectiva el menor. Las figuras 2, 3 y 4 presentan los resultados de las otras universidades, en los que se puede apreciar que la frecuencia de uso de las 
estrategias de aprendizaje coincide en la mayoría de los casos y, al igual que en la Universidad de Ciencias Aplicadas de Ansbach, la estrategia afectiva es la menos usada. Sin embargo, la estrategia social, y a diferencia de los estudiantes de Ansbach, es la más frecuente en las otras tres universidades.

Analizando a los participantes de estas cuatro universidades, vemos que en la Universidad de Ansbach la frecuencia de uso de las estrategias varía con respecto a las otras universidades. En cambio, observamos que al analizar la variable sexo de los participantes, las cuatro universidades presentan los mismos resultados; las estrategias metacognitiva y cognitiva son más frecuentes en hombres que en mujeres mientras que las estrategias mnemotécnica y compensatoria son más usadas por las mujeres. Por lo tanto, encontramos aquí una pequeña diferencia entre los métodos de aprendizajes utilizados por hombres y mujeres. Estos datos están expuestos en los gráficos de las figuras 5, 6, 7 y 8. Las mujeres están representadas en color amarillo y los hombres en azul).

Figura 5. T-Test de frecuencia de uso de categorías entre mujeres y hombres (HS Ansbach).

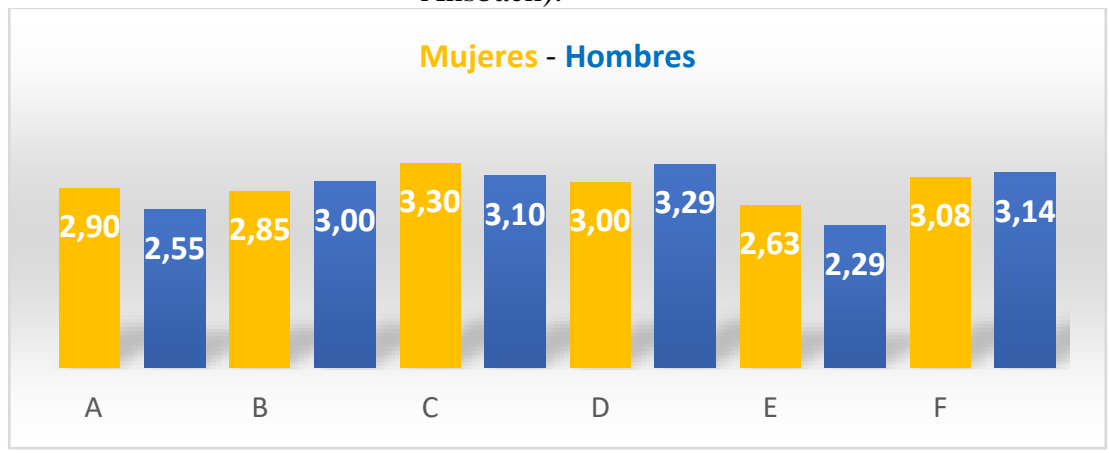

En el caso de la figura 5 podemos apreciar la diferencia entre hombres y mujeres. Los hombres presentan un mayor uso de la estrategia metacognitiva, mientras que las mujeres utilizan más la estrategia compensatoria. Sin embargo, tanto en mujeres como en hombres, el valor medio más bajo revela que la estrategia afectiva es la menos usada. 
María del Carmen Mahúgo Cárdenes

Figura 6. T-Test de frecuencia de uso de categorías entre mujeres y hombres (THN).

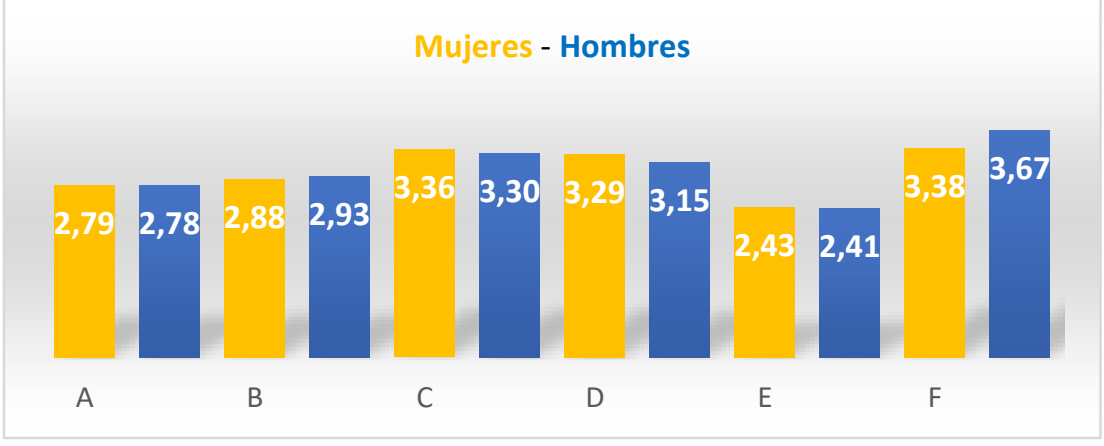

Figura 7. T-Test de frecuencia de uso de categorías entre mujeres y hombres (Universidad de Bayreuth).

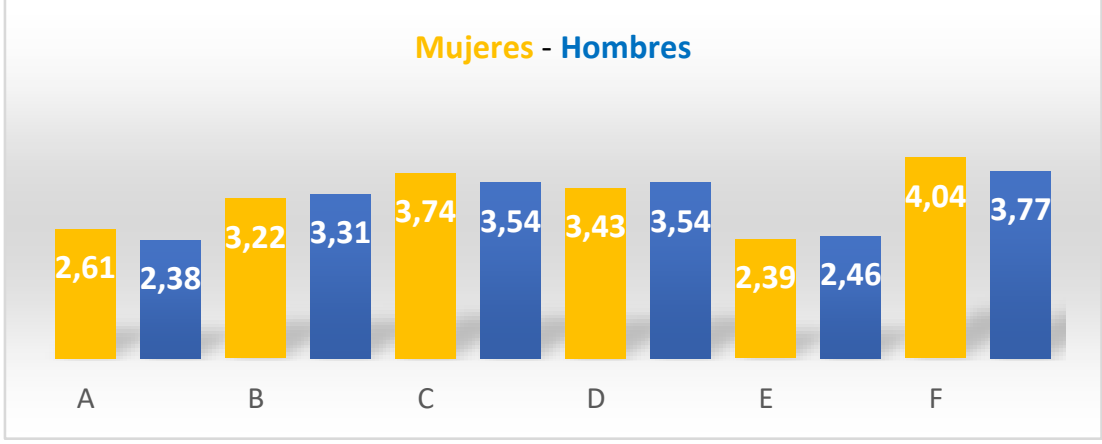

Figura 8. T-Test de frecuencia de uso de categorías entre mujeres y hombres (FAU).

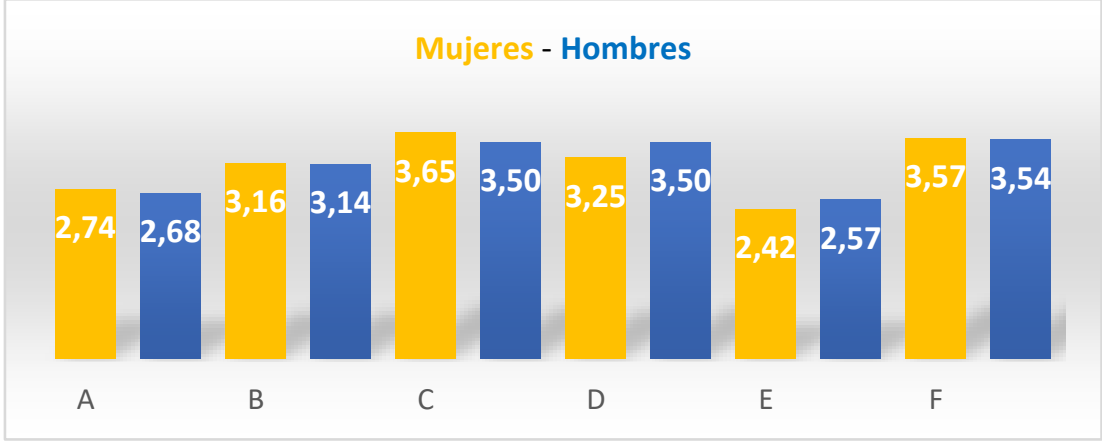


Si observamos los datos de la Universidad de Erlangen (figura 8), advertimos que la similitud entre ambos sexos es mayor que en las otras universidades. Aquí apreciamos cómo en su mayoría, hombres y mujeres utilizan las estrategias de aprendizaje con un porcentaje muy similar.

En cada uno de estos gráficos observamos que la estrategia afectiva presenta el valor medio más bajo en cada una de las universidades, y las estrategias compensatoria, metacognitiva y social alcanza los valores más altos. Además de la frecuencia de uso de las estrategias de aprendizaje y del análisis de la variable Sexo, también hemos indagado en la repercusión que pudieran tener otras variables sobre el uso de las estrategias.

Hemos comparado los datos recabados de las variables L1 y L2 con el uso de las diferentes estrategias de aprendizaje y hemos comprobado mediante una ANOVA si dichas variables están relacionadas con las variables independientes de estrategias mnemotécnica, cognitiva, compensatoria, metacognitiva, afectiva y social. Concretamente, se analizó si la media de cada una de las variables dependientes, L1 y L2 (LE en las tablas) varía según la frecuencia de uso de cada una de las estrategias.

Al aplicar ANOVA de un factor calculamos un test $\mathrm{F}$ y su significación. Este se obtiene al estimar la variación de las medias entre los grupos de la variable independiente y dividirla por la estimación de la variación de las medias dentro de los grupos.

A continuación, las siguientes tablas de datos muestran los resultados del impacto de las variables de L1 y L2 sobre las estrategias de aprendizaje: 
Tabla 1. ANOVA de las variables de estrategias y la variable LE (HS Ansbach).

\begin{tabular}{llrrrrr}
\hline & & $\begin{array}{r}\text { Suma de } \\
\text { cuadrados }\end{array}$ & gl & $\begin{array}{r}\text { Media } \\
\text { cuadrática }\end{array}$ & F & Sig. \\
\hline Mnemotécnica & Inter-grupos &, 105 & 3 &, 035 &, 108 &, 955 \\
& Intra-grupos & 18,734 & 58 &, 323 & & \\
Cognitiva & Inter-grupos & 2,919 & 3 &, 973 & 2,424 &, 074 \\
& Intra-grupos & 24,081 & 60 &, 401 & & \\
Compensatoria & Inter-grupos & 6,683 & 3 & 2,228 & 3,916 &, $013 *$ \\
& Intra-grupos & 32,994 & 58 &, 569 & & \\
Metacognitiva & Inter-grupos & 1,338 & 3 &, 446 &, 798 &, 500 \\
& Intra-grupos & 32,404 & 58 &, 559 & & \\
& & & & & & \\
Afectiva & Inter-grupos & 3,308 & 3 & 1,103 & 2,119 &, 108 \\
& Intra-grupos & 30,192 & 58 &, 521 & & \\
& & & & & & \\
Social & Inter-grupos & 2,785 & 3 &, 928 & 1,315 &, 278 \\
& Intra-grupos & 40,957 & 58 &, 706 & & \\
\hline
\end{tabular}

$* p<0.05$ 
Estrategias de aprendizaje de universitarios en ELE para el sector profesional...

Tabla 2. ANOVA de las variables de estrategias y la variable LE (THN).

\begin{tabular}{llrrrrr}
\hline & & $\begin{array}{r}\text { Suma de } \\
\text { cuadrados }\end{array}$ & gl & $\begin{array}{r}\text { Media } \\
\text { cuadrática }\end{array}$ & F & Sig. \\
\hline Mnemotécnica & Inter-grupos &, 173 & 4 &, 043 &, 119 &, 975 \\
& Intra-grupos & 23,613 & 65 &, 363 & & \\
Cognitiva & Inter-grupos &, 576 & 4 &, 144 &, 495 &, 739 \\
& Intra-grupos & 18,910 & 65 &, 291 & & \\
& & & & & & \\
Compensatoria & Inter-grupos & 7,733 & 4 & 1,933 & 3,963 &, $006^{*}$ \\
& Intra-grupos & 31,710 & 65 &, 488 & & \\
Metacognitiva & Inter-grupos & 3,389 & 4 &, 847 & 2,004 &, 104 \\
& Intra-grupos & 27,482 & 65 &, 423 & & \\
Afectiva & Inter-grupos & 2,193 & 4 &, 548 & 1,082 & \multirow{2}{*}{, 373} \\
& Intra-grupos & 32,950 & 65 &, 507 & & \\
& & & & & & \\
Social & Inter-grupos & 3,783 & 4 &, 946 & 1,065 &, 381 \\
& Intra-grupos & 57,703 & 65 &, 888 & & \\
\hline
\end{tabular}

$*_{p}<0.05$ 
Tabla 3. ANOVA de las variables de estrategias y la variable LE (FAU).

\begin{tabular}{llrrrrr}
\hline & & $\begin{array}{r}\text { Suma de } \\
\text { cuadrados }\end{array}$ & gl & $\begin{array}{r}\text { Media } \\
\text { cuadrática }\end{array}$ & F & Sig. \\
\hline Mnemotécnica & Inter-grupos & 2,229 & 5 &, 446 & 1,086 &, 376 \\
& Intra-grupos & 29,566 & 72 &, 411 & & \\
Cognitiva & Inter-grupos & 4,917 & 5 &, 983 & 2,422 &, $044^{*}$ \\
& Intra-grupos & 29,237 & 72 &, 406 & & \\
& & & & & & \\
Compensatoria & Inter-grupos & 6,414 & 5 & 1,283 & 2,522 &, $037^{*}$ \\
& Intra-grupos & 36,106 & 71 &, 509 & & \\
& & & & & & \\
Metacognitiva & Inter-grupos & 4,234 & 5 &, 847 & 1,435 &, 222 \\
& Intra-grupos & 43,654 & 74 &, 590 & & \\
& & & & & & \\
Afectiva & Inter-grupos & 4,106 & 5 &, 821 & 2,049 &, 081 \\
& Intra-grupos & 30,066 & 75 &, 401 & & \\
& & & & & & \\
Social & Inter-grupos & 5,877 & 5 & 1,175 & 2,093 &, 076 \\
& Intra-grupos & 42,123 & 75 &, 562 & & \\
\hline
\end{tabular}

$* p<0.05$

Como se puede apreciar, en la tabla 1 el valor de F es 3,916 y la significación 0,013; en la tabla 2 el valor de F es 3,963 y la significación 0,006; y en la tabla 3 el valor de F es 1,283 y la significación 0,037. Al ser la significación menor de 0,05 evidencia que las diferencias de media de lenguas extranjeras entre la frecuencia de uso de la categoría compensatoria son significativas. En tal caso, la relación entre L 2 y estrategia compensatoria es mayor con respecto a las otras estrategias. Los resultados obtenidos en la Universidad de Bayreuth no han mostrado ningún valor significativo por lo que consideramos irrelevante mostrar una tabla representativa.

Las siguientes tablas muestran la influencia de la variable L1 en las universidades de Bayreuth y TH Nürnberg Georg Simon Ohm, por lo tanto, 
Estrategias de aprendizaje de universitarios en ELE para el sector profesional...

hemos descartado representar los datos de las otras dos universidades por no mostrar valores significativos.

Tabla 4. ANOVA de las variables de estrategias y la variable L1 (Universidad de Bayreuth).

\begin{tabular}{llrrrrr}
\hline & & $\begin{array}{r}\text { Suma de } \\
\text { cuadrados }\end{array}$ & gl & $\begin{array}{r}\text { Media } \\
\text { cuadrática }\end{array}$ & F & Sig. \\
\hline Mnemotécnica & Inter-grupos & 2,590 & 2 & 1,295 & 4,116 &, $025^{*}$ \\
& Intra-grupos & 10,382 & 33 &, 315 & & \\
Cognitiva & Inter-grupos & 8,132 & 2 & 4,066 & 9,180 &, $001^{*}$ \\
& Intra-grupos & 14,618 & 33 &, 443 & & \\
Compensatoria & Inter-grupos &, 559 & 2 &, 279 & 1,239 & \multirow{2}{*}{, 303} \\
& Intra-grupos & 7,441 & 33 &, 225 & & \\
Metacognitiva & Inter-grupos &, 472 & 2 &, 236 &, 623 &, 542 \\
& Intra-grupos & 12,500 & 33 &, 379 & & \\
Afectiva & Inter-grupos & 2,279 & 2 & 1,140 & 3,016 &, 063 \\
& Intra-grupos & 12,471 & 33 &, 378 & & \\
Social & Inter-grupos &, 918 & 2 &, 459 &, 893 &, 419 \\
& Intra-grupos & 16,971 & 33 &, 514 & & \\
\hline
\end{tabular}

$* p<0.05$ 
Tabla 5. ANOVA de las variables de estrategias y la variable L1 (FAU).

\begin{tabular}{llrrrrr}
\hline & & $\begin{array}{r}\text { Suma de } \\
\text { cuadrados }\end{array}$ & gl & $\begin{array}{r}\text { Media } \\
\text { cuadrática }\end{array}$ & F & Sig. \\
\hline Mnemotécnica & Inter-grupos & 1,498 & 2 &, 749 & 1,835 &, 167 \\
& Intra-grupos & 30,216 & 74 &, 408 & & \\
Cognitiva & Inter-grupos & 4,781 & 2 & 2,390 & 6,174 &, $003^{*}$ \\
& Intra-grupos & 28,648 & 74 &, 387 & & \\
Compensatoria & Inter-grupos & 2,417 & 2 & 1,208 & 2,315 &, 106 \\
& Intra-grupos & 38,110 & 73 &, 522 & & \\
Metacognitiva & Inter-grupos & 3,589 & 2 & 1,795 & 3,110 &, $050^{*}$ \\
& Intra-grupos & 43,854 & 76 &, 577 & & \\
Afectiva & Inter-grupos &, 179 & 2 &, 090 &, 204 &, 816 \\
& Intra-grupos & 33,416 & 76 &, 440 & & \\
& & & & & & \\
Social & Inter-grupos & 10,956 & 2 & 5,478 & 11,363 &, $000^{*}$ \\
& Intra-grupos & 36,639 & 76 &, 482 & & \\
\hline
\end{tabular}

$* p<0.05$

Al estudiar la relación entre L1 y las estrategias de aprendizaje, tablas 4 y 5, observamos que tanto en la Universidad de Bayreuth como en la universidad de Erlangen existe una relación significativa entre estrategia cognitiva y L1. Además, se dan más casos de relación entre L1 y estrategia mnemotécnica en Bayreuth y L1 y estrategias social y metacognitiva en la universidad de Erlangen.

$\mathrm{Si}$ comparamos las cuatro universidades observamos que los resultados difieren en el análisis de las variables L1 y L2 e incluso no hemos hallado significancia en estas variables en algunas de las universidades. Con respecto a la frecuencia de uso y la diferencia entre hombres y mujeres hemos obtenido resultados semejantes en todas las universidades. 
Si hacemos una observación detenida de algunas de las variables y de la frecuencia de uso de algunas categorías, podemos profundizar en las comparaciones entre las variables analizadas. Para empezar, la edad de los estudiantes tiene una media de entre los 21 y 22 años. Esta circunstancia se da debido al sistema educativo del país germano, donde la importancia de un bagaje práctico hace que los estudiantes no comiencen la etapa universitaria directamente después de haber realizado los exámenes de (Abitur). Si continuamos con la variable de la LE, observamos que la media tiene un promedio entre 3 y 4 lenguas extranjeras. En este caso podemos hacer alusión al interés de Alemania en el aprendizaje de lenguas extranjeras además de motivos económicos y estar en contacto geográficamente con muchos otros países.

En la categoría cognitiva hemos advertido que los hombres tienen una media de frecuencia mayor que la de las mujeres y quizá esto se deba a que la mayoría de los hombres estudia carreras del ámbito de la Ingeniería y la Tecnología donde se da un mayor uso del "trabajo cognitivo", "la comprensión profunda, la deducción y el razonamiento, una exigencia de estudio más cualitativo" (MARUGÁN, MARTÍN, CATALINA Y ROMÁN, 2013).

La categoría afectiva ha obtenido en estos análisis un valor minoritario. Al final de los cuestionarios algunos estudiantes manifestaron de forma escrita y oral sus impresiones y muchos coincidieron en un cierto rechazo en la dimensión de esta categoría afectiva. La mayoría no sentía una identificación con esa relación de métodos de aprendizaje. Las estrategias compensatoria y social presentan una media más alta en todos los grupos debido al perfil plurilingüe de los sujetos. Como bien expone Cáceres Lorenzo (2014, p. 229) "la transcendencia que tiene para el universitario el progreso de una conciencia metalingüística se debe entender como el desarrollo del conocimiento explícito de la lengua y del saber aprender (autonomía del alumno) de la competencia discursiva".

En nuestros análisis estadísticos a través de un test bivariado de Pearson no hemos hallado correlaciones significantes entre la variable edad y cada una de las categorías de las estrategias de aprendizaje. Los estudiantes que no tienen el alemán como primera lengua son los que tienen un mayor número de L2.

En este estudio de caso también nos hemos encontrado con algunos inconvenientes a la hora de recabar los datos, por ejemplo, en las informaciones recogidas sobre L1 y L2. Para poder cursar estudios en las 
universidades alemanas los estudiantes procedentes de otros países necesitan un nivel $\mathrm{C} 1$ de alemán, por lo que se parte de que todos los encuestados que no son de origen alemán poseen este nivel, aunque por descuido o equivocación no lo hayan dejado constante en el apartado de preguntas personales del cuestionario de estrategias de aprendizaje utilizado para este estudio.

\section{Consideraciones finales}

Los datos recogidos y analizados en esta investigación nos permiten responder a nuestras preguntas de investigación:

(1) ¿Cómo se definen las estrategias de aprendizaje de los estudiantes universitarios de ELE en Alemania?

En los datos descritos en este artículo hemos mostrado qué características y similitudes existen entre estos grupos de universitarios en el uso de estrategias de aprendizaje que utilizan y las variables que caracterizan a dichos sujetos y por lo tanto consideramos que las estrategias de aprendizaje de los estudiantes universitarios de ELE en Alemania se definen como método fundamental en su aprendizaje autónomo del español. Los resultados obtenidos a través de los cuestionarios verifican que los estudiantes utilizan estrategias de aprendizaje en el proceso de adquisición de una LE en una frecuencia de uso media-alta.

(2) ¿Cuál o cuáles son las estrategias de aprendizaje predominantes?

Las estrategias que presentan una mayor frecuencia de uso son la compensatoria y social presentando ambas una frecuencia de uso alto, mientras que las estrategias menos utilizadas son las de la categoría afectiva. Los resultados indican que los aprendices tienden a aplicar por este orden las distintas categorías: compensatorias, socioculturales, metacognitivas, cognitivas, mnemotécnicas, y afectivas.

Por lo tanto nuestros sujetos de investigación consideran importantes estrategias como intentar adivinar el significado de las palabras extrañas, inventar palabras cuando se está bloqueado, leer sin buscar el significado de todas las palabras, intentar adivinar lo que otra persona dice, usar sinónimos para expresarse, pedir que le corrijan cuando hablan, practicar el español con otros estudiantes, solicitar ayuda de los profesores, hacer preguntas en español, intentar comprender la cultura hispana, prestar atención al hablante cuando está hablando español, anotar los errores y planificar el tiempo de 
estudio, ver la tele, series y escuchar música en español, intentar encontrar normas o reglas que le faciliten el estudio y utilizar fichas para recordar nuevas palabras.

(3) ¿Existe alguna variable personal que influya en el uso de las estrategias?

Asimismo, existen condiciones, ya sean internas o externas, del aprendizaje de las que depende la calidad de dichos procesos autónomos de aprendizaje y de los resultados del mismo. Las variables sexo, L1 y L2 influyen en las decisiones autónomas que los aprendientes toman en el momento de asumir una determinada tarea de aprendizaje y utilizar una estrategia puede afectar de manera significativa a la adquisición de la lengua meta en cuestión.

El uso de estrategias de aprendizaje es muy positivo para el desarrollo autónomo de los aprendientes. A través de este estudio ha sido posible analizar las estrategias de estos estudiantes y nos ayuda, como profesores de español como lengua extranjera a mejorar el potencial de aprendizaje y trabajo de nuestros estudiantes. Este estudio nos muestra también las áreas de estilo que no se usan demasiado y por consiguiente poder trabajar en el desarrollo de esas áreas.

Llevar a cabo actividades que no siempre son las que más se ajustan a las preferencias de aprendizaje de nuestros estudiantes a menudo ayuda a romper con la monotonía y a subsanar errores en el aprendizaje de la LE. Si, por ejemplo, nuestros estudiantes no utilizan la estrategia afectiva, se produce una carencia en el aprendizaje de la LE y por lo tanto debemos actuar como mediadores para que los alumnos desarrollen la motivación, una de las principales características de la estrategia afectivas.

Creemos que las competencias lingüísticas en universitarios que aprenden español como lengua extranjera se desarrollan si los convertimos en estudiantes autónomos, por lo tanto, es necesario el análisis del proceso de enseñanza/aprendizaje del español como lengua extranjera y averiguar el impacto de las variables y la influencia de otros métodos de aprendizaje ya aprendidos en el proceso de enseñanza/aprendizaje.

Resolver este problema de investigación contribuye al conocimiento de EAL de los aprendientes de ELE europeos y ayuda a mejorar las habilidades lingüísticas de los alumnos en el aprendizaje de la lengua meta y su necesario desarrollo como aprendiz independiente. 


\section{Referencias bibliográficas}

ALCALDE MATO, N. Orígenes y desarrollo de la enseñanza de lenguas extranjeras en Alemania. Pragmalinguiística, v, 18, p. 8-29, 2010.

ALCALDE MATO, N. Principales métodos de enseñanza de lenguas extranjeras en Alemania. Revista de Lingüística y Lenguas Aplicadas, v. 6, p. 9-23, 2011.

BIMMEL, P. Lernstrategien: bausteine der lernerautonomie. Deutsch als Fremdsprache, Heft 46, p. 3-10. München: Hueber. 2012.

CÁCERES LORENZO, M. T. La rúbrica de un taller de escritura para enseñar a sinohablantes en el contexto universitario europeo: estudio de caso. Porta Linguarum, v. 21, p. 227-244, 2014.

CENTRO VIRTUAL CERVANTES. Estrategias de aprendizaje. Diccionario de términos clave de ELE. Recuperado el 10 de mayo de 2019, de

https://cvc.cervantes.es/ensenanza/biblioteca_ele/diccio_ele/diccionario/est rategias.htm

COHEN, A. Language Learning: insights for learners, teachers, and researchers. New York: Newbury House. 1990.

EHRMAN M. L.; OXFORD R. L.. Adult language learning styles and strategies in an intensive training setting. Modern Language Journal, v. 74, n. 3, p. 311-327, 1990.

FELD-KNAPP, I. Autonomie/autonomes Lernen. En H. BARKOWSKI; H.-J. KRUMM (Eds.). Fachlexikon Deutsch als Fremd- und Zweitsprache. Tübingen/Basel: Francke, p. 21. 2010.

FERNÁNDEZ-CASTILLO, A. Estrategias de aprendizaje y adquisición de una segunda lengua. Reidocrea, v. 4, n. 48, p. 391-404, 2015. 
Estrategias de aprendizaje de universitarios en ELE para el sector profesional...

FRAILE ARANDA, A. El sistema universitario europeo como modelo posible para la educación superior latinoamericana. Revista Electrónica de Investigación Educativa, v. 8, n. 1. 2006.

GALINDO MERINO, M. M. La lengua materna en el aula de ELE. Colección monografías de ASELE, v. 15. Málaga. 2012. Recuperado el 15 de mayo de 2019, de

http://www.cervantesvirtual.com/obra/la-lengua-materna-en-el-aula-de-ele/

GARCÍA COBO, A. Variables afectivas en la adquisición de una lengua extranjera: motivación y ansiedad. 2013. Recuperado el 20 de enero de 2019, de https://repositorio.unican.es/xmlui/handle/10902/3972

GARCÍA HERRERO, M. M. Diferencias en el uso de estrategias en el aprendizaje de la lengua extranjera según el género. Docencia e Investigación, v. 37, p. 61-80, 2012.

GARCÍA HERRERO, M. M. Utilización de estrategias y selección de estilos por estudiantes universitarios, ya titulados, en el aprendizaje de la lengua extranjera. Educar, v. 49, n. 1, p. 127-151, 2013.

GREEN, J.; OXFORD R. L.. A closer look at learning strategies, L2 proficiency, and gender. TESOL Quarterly, v. 29, p. 261-297, 1995.

GRIFFITHS, C. Strategies and good language learners. En C. GRIFFITHS (Eds.) Lessons from good language learners. New York: Cambridge University Press, 2008. p. 83-98.

GRÜNEWALD, A. La motivación de los alumnos en la clase de lengua extranjera. Resultados de una investigación empírica en el contexto del uso de las tecnologías de comunicación e información. Pulso, v. 32, p. 75-94, 2009.

LASAGABASTER, D. El papel del inglés en el fomento del multilingüismo en la universidad. ELIA, v. 12, p. 13-44, 2012. 
MARUGÁN, M., MARTÍN, L. J.; CATAlinA, J.; ROMÁN, J. M. Estrategias cognitivas de elaboración y naturaleza de los contenidos en estudiantes universitarios. Psicología Educativa, v. 19, p. 13-20, 2013.

MUÑOZ ZAYAS, R. El aprendizaje de lenguas extranjeras en España. eXtoikos, v. 9, p. 63-68, 2013.

MUÑOZ, C. Aprender Idiomas. Barcelona: Ediciones Paidós, 2002.

O’MALEY, M.; CHAMOT, A.. Learning strategies in language acquisition. Londres: Cambridge University Press, 1990.

OLMOS SERRANO, I. El español en el mundo: anuario del Instituto Cervantes: el español en Alemania por Ignacio Olmos Serrano, 258. Arco Libros, s.1., 2006. Recuperado el 17 de abril de 2019, de https://cvc.cervantes.es/lengua/anuario/anuario_06-07/pdf/paises_54.pdf

OXFORD, R. Language learning strategies: what every teacher should know. New York, NY: Newbury House, 1990.

RONCEL VEGA, V. M. Variables explicativas del rendimiento académico en una LE. Redele, v. 4, 2005.

SÁNCHEZ CASTRO, M. Dificultades de los hablantes nativos de alemán en el aprendizaje de los tiempos de pasado. Redele, v. 13, p. 1-10, 2008.

\section{Apéndice}

Strategien zum Erlernen der spanischen Sprache Bitte beantworten Sie die folgenden Fragen:

1. Alter

2. Geschlecht

männlich weiblich

3. Studiengang

4. Name der Universität / Hochschule

5. Spanischkenntnisse 
Estrategias de aprendizaje de universitarios en ELE para el sector profesional...

Niveau: $\square$ A0

A1

B1

$\neg \mathrm{C} 1$
A2
B2

C2

Elementare Sprachverwendung (A1 und A2)

Selbstständige Sprachverwendung (B1 und B2)

Kompetente Sprachverwendung (C1 und C2)

6. Wie lange haben Sie Spanisch gelernt?
Weniger als 6 Monate
$\square$ Ein Semester $\square$ Zwei Semester
Länger als ein Jahr
Drei oder mehr Jahre

7. Haben Sie ein Sprachzertifikat für Spanisch als Fremdsprache?

Ja $\quad \square$ Nein

Wenn JA, welche(s)? (DELE, TELC, o.ä.)

Zertifikat

Niveau

Zertifikat

Niveau

8. Andere Sprachkenntnisse (A1, A2, B1, B2, C1, C2, Muttersprachler)

Sprache

Sprache

Niveau

Niveau

Sprache

Niveau

Weitere:

9. Welche Ziele sind Ihnen wichtig?

Sprachkenntnisse für den privaten Alltag erwerben oder erweitern.

Sprachkenntnisse für den beruflichen Alltag erwerben oder erweitern.

Andere:

Markieren Sie von 1 bis 5 Ihre Zustimmung:

1. Trifft nicht zu

2. Trifft selten $\mathrm{zu}$

3. Trifft manchmal/teilweise $\mathrm{zu}$

4. Trifft oft/meistens $\mathrm{zu}$

5. Trifft immer zu 


\section{Abschnitt: A}

1. Ich denke über die Zusammenhänge zwischen dem, was ich bereits weiß, und neuen Dingen, die ich in Spanisch lerne, nach.

2. Ich verwende neue spanische Wörter in einem Satz, um sie mir besser zu merken.

3. Ich verbinde den Klang eines spanischen Wortes mit einem Bild, damit ich mich besser daran erinnern kann.

4. Ich erinnere mich an ein neues spanisches Wort, indem ich mir dieses in einer Situation bildlich vorstelle.

5. Ich benutze Reime, damit ich mir spanische Wörter merken kann.

6. Ich verwende Lernkarten, um neue spanische Wörter zu lernen.

7. Ich stelle neue Wörter schauspielerisch dar.

8. Ich gehe die Lektionen im Lehrbuch zu Hause nochmal durch.

9. Ich erinnere mich an neue Wörter oder

Redewendungen, wenn ich mich zum Beispiel an ihren

Platz auf einer Buchseite, der Tafel oder einem

Straßenschild erinnere.

\section{Abschnitt: B}

1. Ich spreche oder schreibe neue spanische Wörter mehrmals.

2. Ich versuche, wie Spanisch-Muttersprachler zu sprechen.

3. Ich übe die spanische Aussprache.

4. Ich verwende ein spanisches Wort, das ich kenne, in verschiedenen Situationen.

5. Ich beginne Gespräche auf Spanisch.

6. Ich sehe mir spanische Fernsehsendungen oder spanische Filme an.

7. Ich lese in der Freizeit spanische Bücher, Zeitungen.

8. Ich schreibe Notizen, Nachrichten, Briefe oder

Berichte auf Spanisch. 
9. Zunächst überfliege ich einen spanischen Text, bevor ich ihn genauer lese.

10. Ich suche nach Wörtern in meiner Muttersprache, die neuen spanischen Vokabeln ähnlich sind.

11. Ich versuche, Gesetzmäßigkeiten im Spanischen zu erkennen.

12. Ich finde die Bedeutung von spanischen Wörtern heraus, indem ich sie in verschiedene Teile zerlege, die ich verstehe.

13. Ich vermeide, Wort für Wort zu übersetzen.

14. Ich fasse Informationen zusammen, die ich auf Spanisch höre oder lese.

\section{Abschnitt: C}

1. Ich versuche, die Bedeutung einer mir unbekannten Vokabel zu erraten.

2. Wenn mir ein Wort im Gespräch fehlt, drücke ich es mit Gesten aus.

3. Ich erfinde neue Wörter, wenn mir die richtigen im Spanischen fehlen.

4. Ich lese spanische Texte, ohne jedes unbekannte Wort nachzuschlagen.

5. Ich versuche zu erraten, was der Gesprächspartner als nächstes auf Spanisch sagen möchte.

6. Wenn mir eine spanische Vokabel nicht einfällt, benutze ich ein ähnliches Wort oder einen Satz, um diese Vokabel zu umschreiben.

\section{Abschnitt: D}

1. Ich versuche meine Spanischkenntnisse so vielfältig wie möglich anzuwenden.

2. Ich achte auf meine Fehler im Spanischen und lerne aus ihnen.

3. Ich höre aufmerksam zu, wenn jemand Spanisch spricht.

4. Ich versuche herauszufinden, wie ich besser Spanisch lernen kann. 
5. Ich richte mir meinen Tagesablauf so ein, dass mir genug Zeit zum Spanisch lernen bleibt.

6. Ich suche Leute, mit denen ich Spanisch sprechen kann.

7. Ich suche nach Gelegenheiten, so viel wie möglich spanische Texte zu lesen.

8. Ich habe klare Ziele, was die Verbesserung meiner Spanischkenntnisse angeht.

9. Ich denke über meine Fortschritte beim Erlernen der spanischen Sprache nach.

\section{Abschnitt: E}

1. Wenn ich Angst habe, Spanisch zu sprechen, versuche ich, mich bewusst zu entspannen.

2. Ich ermutige mich, Spanisch zu sprechen, auch wenn ich Angst vor Fehlern habe.

3. Ich belohne mich, wenn ich Lernfortschritte im Spanischen mache.

4. Ich merke, ob ich angespannt oder nervös bin, wenn ich Spanisch lerne.

5. Ich führe ein Lerntagebuch und schreibe meine Gefühle beim Lernen auf.

6. Ich spreche mit anderen darüber, wie ich mich beim Spanischlernen fühle.

\section{Abschnitt: F}

1. Wenn ich etwas auf Spanisch nicht verstehe, bitte ich den Gesprächspartner, langsamer zu sprechen oder es zu wiederholen.

2. Ich bitte Muttersprachler, meine Fehler beim

Sprechen zu korrigieren.

3. Ich übe Spanisch mit anderen Studenten.

4. Ich bitte Muttersprachler um Hilfe.

5. Ich stelle Fragen auf Spanisch.

6 . Ich versuche, etwas über die Kultur der spanisch

sprechenden Menschen zu lernen. 
Estrategias de aprendizaje de universitarios en ELE para el sector profesional... 10. Finden Sie diesen Fragebogen zweckmäßig?

11. Würden Sie etwas ändern?

12. Würden Sie weitere Fragen hinzufügen?

Recibido en: 04/09/2019

Aceptado en: 17/03/2020

Title: Learning strategies of university students in Spanish as a foreign language for the professional sector: case study in Germany

Título: Estratégias de aprendizagem de universitários em espanhol como língua estrangeira para o setor profissional: um estudo de caso na Alemanha 